\title{
Travelling Waves for Gas-Solid Reactions
}

\author{
C.J. VAN DUIJN and A. STRAATHOF
}

\begin{abstract}
Bounded travelling waves, arising in a combustion model for gas-solid reactions in a porous medium, are studied. We consider the existence, uniqueness and several qualitative properties. In particular we investigate waves with finiteness and we derive estimates in the limit of vanishing diffusion.
\end{abstract}

\section{INTRODUCTION}

In this paper we study travelling wave solutions of a model describing the combustion (or conversion) of a porous solid as it reacts irreversibly and isothermally with a gas moving through its pores. We shall assume that the reaction is distributed throughout the solid with a rate of reaction proportional to $F(S) G(C)$ where $S$ and $C$ are solid and gas concentrations and $F$ and $G$ positive non-decreasing functions such that $F(0)=G(0)=0$. The structural change of the porous medium during combustion is neglected. Problems of this nature arise in chemical engineering, and an jmportant example is coal gasification, see Froment and Bischoff [FB].

1991 Mathematics Subject Classification: 35 K57, 34 B15.

Editorial Complutense. Madrid, 1994. 
In one space dimension, the mass balance for the solid and the gas yields the equations

$$
\begin{aligned}
S_{t} & =-\lambda_{1} F(S) G(C) \\
\epsilon C_{t}+q C_{x}-D C_{x x} & =-\lambda_{2} F(S) G(C)
\end{aligned}
$$

for $-\infty<x<+\infty$ and $t>0$. Here the positive constants $\epsilon, q, D, \lambda_{1}$ and $\lambda_{2}$ denote the porosity, gas flux, effective diffusivity and the reaction rates, respectively. The underlying gas transport is stationary and directed from $x=-\infty$ (inlet) to $x=+\infty$ (outlet). A derivation of this model was given by Szekely, Evans and Sohn [SES]. Equations (1.1) without convection (i.e. $q=0$ ) were studied by Díaz and Stakgold [DS], Stakgold [S] and Di Liddo, Maddalena and Stakgold [LMS].

In this paper we are interested in travelling wave solutions of equations (1.1). That is, we look for solutions depending on the combination $x-c t$ only, where $c$ is the a priori unknown wavespeed. They satisfy the boundary conditions

$$
S(-\infty, t)=0, \quad C(-\infty, t)=C^{*}>0 \quad t>0
$$

at the inlet, and

$$
S(+\infty, t)=S^{*}>0, \quad C(+\infty, t)=0 \quad t>0
$$

at the outlet, where $S^{*}$ and $C^{*}$ are constants.

The methods used in this paper are primarily developed by Van Duijn and Knabner [vDK] for a system of equations describing nonequilibrium multiple-site adsorption in the transport of reactive solutes through porous media.

Travelling wave solutions for (1.1) without convection lead to unbounded waves. These were investigated by Di Liddo, Maddalena and Stakgold [LMS].

In section 2 we investigate the existence and uniqueness of travelling waves for classes of $F$ and $G$ large enough to cover all power law reactions. 
One has to take into account that $F$ or $G$ may not be Lipschitz continuous at $S=0$ or at $C=0$, respectively. This possible degeneration of the problem may lead to the existence of regions where full conversion or no conversion takes place. Section 3 contains a characterization of these situations. It leads to finite travelling waves.

In section 4 we study the hyperbolic limit of (1.1): i.e. we consider the case of vanishing diffusivity. We identify the limit solution for $D=0$ and give an explicit upperbound for the rate of convergence.

Before proceeding we scale the variables according to:

$$
\begin{aligned}
u & :=\frac{S^{*}-S}{S^{*}}, \quad v:=\frac{C}{C^{*}}, \\
f(y) & :=\lambda_{1} F\left(y S^{*}\right) \quad \text { and } \quad g(y):=G\left(y C^{*}\right) .
\end{aligned}
$$

Then equations (1.1) reduce to

$$
\begin{aligned}
u_{t} & =\frac{1}{S^{*}} f(1-u) g(v) \\
\epsilon v_{t}+q v_{x}-D v_{x x} & =-\frac{\lambda}{C^{*}} f(1-u) g(v)
\end{aligned}
$$

for $-\infty<x<+\infty$ and $t>0$, where $\lambda:=\lambda_{2} / \lambda_{1}$ is the Thiele Modulus. The boundary conditions become

$$
u(-\infty, t)=1, \quad v(-\infty, t)=1 \quad t>0
$$

and

$$
u(+\infty, t)=0, \quad v(+\infty, t)=0 \quad t>0 .
$$

Looking for travelling wave solutions means that we have to determine functions

$$
u=u(\eta), \quad v=v(\eta) \quad \text { with } \eta:=x-c t
$$


and the wavespeed $c$ satisfying

$$
\begin{gathered}
-c u^{\prime}=\frac{1}{S^{*}} f(1-u) g(v) \\
-c\left(\epsilon v+\lambda \frac{S^{*}}{C^{*}} u\right)^{\prime}+q v^{\prime}-D v^{\prime \prime}=0
\end{gathered}
$$

for $-\infty<\eta<+\infty$, where the primes denote differentiation with respect to $\eta$, and

$$
(B C) \begin{cases}u(-\infty)=1, & v(-\infty)=1 \\ u(+\infty)=0, & v(+\infty)=0\end{cases}
$$

Throughout this paper we assume

$$
\text { (H) }\left\{\begin{array}{l}
(i) f, g: \mathbf{R}_{0}^{+} \rightarrow \mathbf{R}_{0}^{+} \text {such that } f, g \in C\left(\mathbf{R}_{0}^{+}\right) \cap C^{0,1}\left(\mathbf{R}^{+}\right) ; \\
(i i) f(0)=g(0)=0, \text { and } f(s)>0, g(s)>0 \text { for } s>0 .
\end{array}\right.
$$

As a first result we have the following

Proposition 1.1. Suppose $\{u, v, c\}$ is a travelling wave for $(B C)$. Then

(i) $u^{\prime}( \pm \infty)=0, \quad v^{\prime}( \pm \infty)=0$;

(ii) $c=\frac{C^{*}}{\epsilon C^{*}+\lambda S^{*}} q$.

Proof. Equation (1.5a), the boundary conditions (BC) and hypothesis $H(i i)$ imply $u^{\prime}( \pm \infty)=0$. Integrating (1.5b) yields

$$
D v^{\prime}=(q-\epsilon c) v-c \lambda \frac{S^{*}}{C^{*}} u+A \quad \text { in } \mathbf{R}
$$

where $A \in \mathbf{R}$ is the constant of integration. Letting $\eta \rightarrow \pm \infty$ we obtain

$$
D v^{\prime}(-\infty)=q-c\left(\epsilon+\lambda \frac{S^{*}}{C^{*}}\right)+A
$$


and

$$
D v^{\prime}(+\infty)=A \text {. }
$$

Clearly $v$ can only satisfy the boundary conditions if these limits are zero. This implies the first assertion. Consequently $A=0$, and the expression for $c$ results from (1.8).

As a direct consequence of $(1.6),(1.7)$ and $A=0$ we have the equivalent formulation of a travelling wave as a first order system, consisting of (1.5a) and (1.7).

Corollary 1.2. A triple $\{u, v, c\}$ with $u, v: \mathbf{R} \rightarrow \mathbf{R}_{0}^{+}$and $c>0$ is a travelling wave for $(B C)$ iff

$$
\begin{aligned}
\text { (i) } u, v & \in C^{\mathbf{1}}(\mathbf{R}) \\
\text { (ii) } u^{\prime} & =-\frac{1}{c S^{*}} f(1-u) g(v) \\
v^{\prime} & =\frac{\lambda c S^{*}}{D C^{*}}(v-u)
\end{aligned}
$$

(iii) $u$ and $v$ satisfy (BC),

where $c$ is defined by $(1,6)$.

Remark 1.3 (i) The wavespeed $c$ can be written as

$$
c=\frac{1}{1+\frac{\lambda S^{*}}{\epsilon C^{*}}} \frac{q}{\epsilon}
$$

which shows that $c<q / \epsilon$.

(ii) In general the methods used in this paper also hold for the boundary conditions

$$
S(-\infty, t)=S_{*}, \quad C(-\infty, t)=C^{*} \quad t>0
$$

and

$$
S(+\infty, t)=S^{*}, \quad C(+\infty, t)=C_{*}, \quad t>0,
$$


with $S^{*}>S_{*}>0$ and $C^{*}>C_{*}>0$.

(iii) From (1.9b) it follows that $v \in C^{2}(\mathbf{R})$.

\section{EXISTENCE AND UNIQUENESS}

In the first part of this section we prove existence of a travelling wave consisting of monotone functions $u$ and $v$. This is done by demonstrating how to construct a connecting orbit in the phaseplane $(u, v)$ between the equilibrium points $(1,1)$ and $(0,0)$.

We start from the formulation given in Corollary 1.2. In the system of equations given there, we reverse the dependent variable by setting

$$
\eta:=-\eta
$$

This leads to

$$
\begin{aligned}
& u^{\prime}=c_{1} f(1-u) g(v) \\
& v^{\prime}=c_{2}(u-v)
\end{aligned}
$$

in $\mathbf{R}$, where $c_{1}=\frac{1}{c S^{*}}$ and $c_{2}=\frac{\lambda c S^{*}}{\partial C^{*}}$. Because we restrict ourselves to monotone waves, the connecting orbit cannot leave the square $[0,1]$ $\times[0,1]$ and in particular $v \leq u$ (from $(2.1 \mathrm{~b})$ ). If we define the set

$$
S:=\{(u, v) \mid 0<v<u<1\}
$$

we must look for a connecting orbit in $\bar{S}$. The boundary of this set consists of the segments $\partial S_{i}, i=1,2,3$, where

$$
\begin{gathered}
\partial S_{1}=\{(u, 0) \mid 0<u<1\} \\
\partial S_{2}=\{(1, v) \mid 0<v<1\}
\end{gathered}
$$

and

$$
\partial S_{3}=\{(u, u) \mid 0<u<1\}
$$


Theorem 2.1. Let hypotheses $H(i)-(i i)$ be satisfied. Then there exists a monotone travelling wave with the properties

(i) $u^{\prime} \leq 0$ in $\mathbf{R}$ and $u^{\prime}<0$ in $\{\eta \in \mathbf{R} \mid u(\eta) \in(0,1)\}$

(ii) $v^{\prime} \leq 0$ in $\mathbf{R}$ and $v^{\prime}<0$ in $\{\eta \in \mathbf{R} \mid v(\eta) \in(0,1)\}$;

(iii) $0 \leq u \leq 1$ and $0 \leq v<1$ in $\mathbf{R}$.

Proof. We give the proof in terms of equations (2.1), thus in the reversed coordinate. To be specific we construct an orbit in $S \cup \partial S_{2}$ which connects the equilibrium points $(0,0)$ and $(1,1)$. This is done in two steps.

1. There is a negative half orbit in $S$ leaving $(0,0)$.

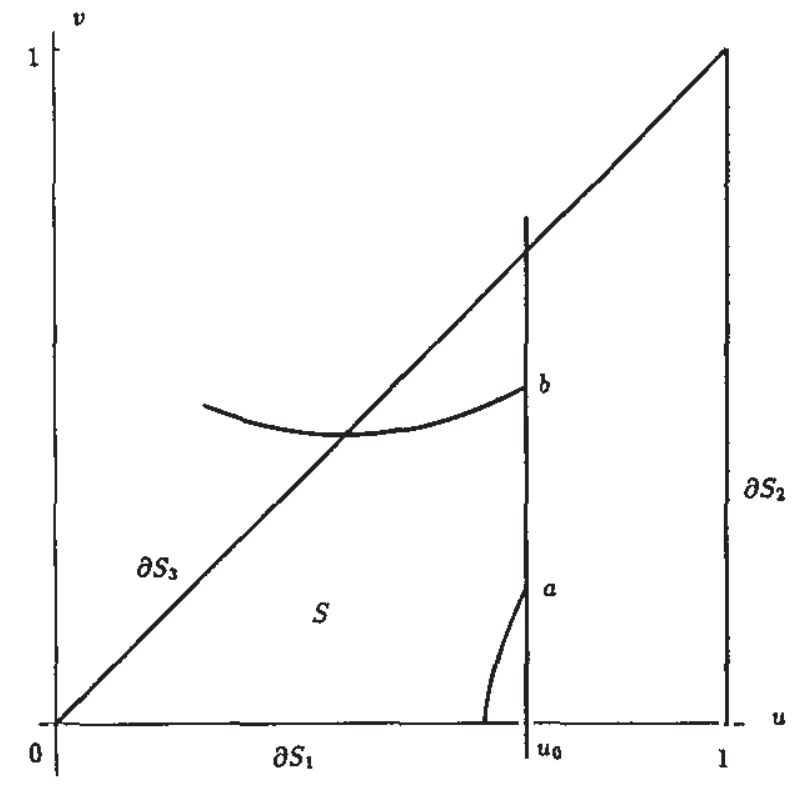

Fig. 1. The shooting argument in the phaseplane $(u, v)$.

We use a shooting argument. Choose $u_{0} \in(0,1)$. In the interval $\left(0, u_{0}\right)$ on the $v$-axis we consider two subsets $A$ and $B$. These are characterized as follows. A point $a \in\left(0, u_{0}\right)$ belongs to the set $A$ if the negative half orbit which starts at $\left(u_{0}, a\right)$ has a point of $\partial S_{1}$ as exit point, see 
Figure 1. Since $u^{\prime}(\eta)>0, v^{\prime}(\eta)>0$ as long as $(u(\eta), v(\eta)) \in S$ this is well defined. A has the following properties:

(i) $A$ is nonempty by the following argument: every half orbit $(u, v)$ starting at $(\tilde{u}, 0)$ with $0<\tilde{u}<u_{0}$ must be in $S$ for small times. Because $v$ is strictly increasing in $S$ the orbit cannot leave through the boundary $\partial S_{1}$. Next suppose the orbit leaves through $\partial S_{3}$ for $\eta=\tilde{\eta}$. Clearly $v^{\prime}(\tilde{\eta})=0$ which implies that for $\eta<\tilde{\eta}$, sufficiently close, the solution must be above $\partial S_{3}$, a contradiction.

(ii) If $a_{1} \in A$, then there exists $a_{2}>a_{1}$ with $a_{2} \in A$. The argument is similar. (Note that orbits in $S$ do not intersect.)

Set $\bar{a}=\sup A$. Clearly $\bar{a} \notin A$. Similarly a point $b \in\left(0, u_{0}\right)$ belongs to the set $B$ if the negative half orbit which starts at $\left(u_{0}, b\right)$ has a point of $\partial S_{3}$ as exit point. Again we can show that (i) $B$ is nonempty and (ii) if $b_{1} \in B$ then there exists $b_{2}<b_{1}$ with $b_{2} \in B$. We now set $\bar{b}=\inf B$. Clearly $\bar{b} \notin B$. From the definition one observes that $\bar{a} \leq \bar{b}$. Choose $d \in[\bar{a}, \bar{b}]$. Now consider the negative half orbit $\{(u(\eta), v(\eta)) \mid \eta<0\}$ which starts at $\left(u_{0}, d\right)$. Define $\underline{\eta} \in[-\infty, 0)$ as

$$
\underline{\eta}=\inf \{\eta<0 \mid(u(\eta), v(\eta)) \in S\} .
$$

Clearly $(u(\underline{\eta}), v(\underline{\eta}))=(0,0)$. We distinguish the following possibilities:

- $\underline{\eta}=-\infty$. This gives the desired half orbit on $\mathbf{R}^{-}$.

- $\underline{\eta}>-\infty$. This is possible if the problem degenerates at $(0,0)$. We continue the solution as $(u(\eta), v(\eta))=(0,0)$ for $\eta<\underline{\eta}$. Note that the solution remains differentiable.

2. To complete the proof we have to connect the points $\left(u_{0}, d\right)$ and $(1,1)$. As long as $(u, v) \in S$ we can continue the orbit. Define

$$
\ddot{\eta}=\sup \{\eta>0\}(u(\eta), v(\eta)) \in S\}
$$

First note that $(u(\eta), v(\eta))$ cannot leave through $\partial S_{3}$ by the same argument as above. Again we distinguish the following possibilities:

- $\bar{\eta}=+\infty$. This gives the desired orbit on $\mathbf{R}$. 
- $\vec{\eta}<+\infty$. If $(u(\bar{\eta}), v(\bar{\eta})) \in \partial S_{2}$ we continue the orbit as $(1, v(\eta))$ for $\eta>\bar{\eta}$ by the (unique) solution of

$$
v^{\prime}=c_{2}(1-v)
$$

with initial value $v(\bar{\eta})$. The solution is given by

$$
v(\eta)=1-(1-v(\bar{\eta})) \exp \left(-c_{2}(\eta-\bar{\eta})\right)
$$

Note that such a solution is again differentiable in $\eta=\bar{\eta}$.

The remaining possibility $(u(\bar{\eta}), v(\bar{\eta}))=(1,1)$ cannot occur by the following argument: suppose $(u(\bar{\eta}), v(\bar{\eta}))=(1,1)$ for $\bar{\eta}<+\infty$. Solving equation $(2.1 \mathrm{~b})$ on $(0, \bar{\eta})$ gives

$$
v(\bar{\eta}) e^{c_{2} \tilde{\eta}}-v(0)=c_{2} \int_{0}^{\bar{\eta}} u(\eta) e^{c_{2} \eta} d \eta
$$

or

$$
e^{c_{2} \bar{\eta}}-v(0) \leq c_{2} \int_{0}^{\bar{\eta}} e^{c_{2} \eta} d \eta=e^{c_{2} \bar{\eta}}-1
$$

which leads to $v(0) \geq 1$, a contradiction. This proves assertion (iii).

In Figure 2 we show an example of an orbit in the phase plane and the corresponding solutions $u, v$ in the original independent variable.

Remark 2.2. (i) Theorem 2.1 implies the existence of a classical solution $u \in C^{1}(\mathbf{R}), v \in C^{2}(\mathbf{R})$ of equations (1.5) for (BC), or in terms of $x$ and $t$ of Problem (1.3), (1.4).

(ii) If $f \in C^{0,1}\left(\mathbf{R}_{0}^{+}\right)$, then also $u<1$ in $\mathbf{R}$.

(iii) If $g \in C^{0,1}\left(\mathbf{R}_{0}^{+}\right)$, then $u, v>0$ in $\mathbf{R}$.

Next we consider the uniqueness for travelling waves. We introduce and additional hypothesis on $f$ and $g$ :

$H($ iii) $f$ and $g$ are monotone non-decreasing functions. 

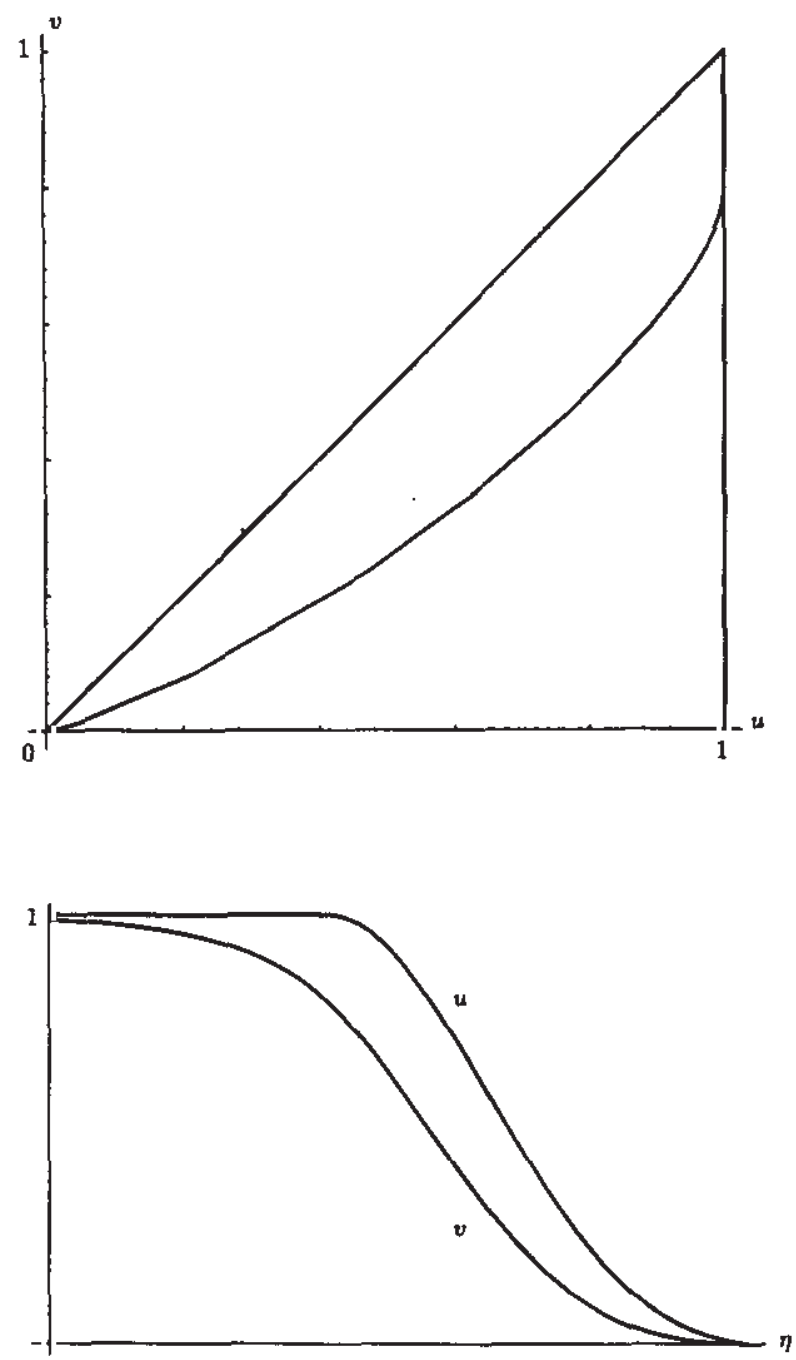

Fig. 2. Example of an orbit in the phase-plane and the solutions $u, v$ fór $f(s)=g(s)=s^{1 / 2}$ (i.e. both degenerate), $C^{*}=1, S^{*}=1, \lambda=$ $3 / 2, \epsilon=1 / 2, q=2, D=3 / 2$. 
Due to the following theorem we have uniqueness up to translation.

Theorem 2.3. Let $f$ and $g$ satisfy $H(i)$-(iii). Suppose there exist two travelling waves $\left\{u_{i}, v_{i}, c\right\}, i=1,2$, which satisfy the same boundary conditions (BC) and are such that $0 \leq v_{i} \leq u_{i} \leq 1$ in $\mathbf{R}$ for $i=1,2$. Then there exists an $\eta_{0} \in \mathbf{R}$ such that

$$
u_{1}(\cdot)=u_{2}\left(\cdot+\eta_{0}\right) \quad \text { and } \quad v_{1}(\cdot)=v_{2}\left(\cdot+\eta_{0}\right) \quad \text { in } \mathbf{R} \text {. }
$$

Remark 2.4. Because we prove here uniqueness in a class of travelling waves for which $0 \leq v \leq u \leq 1$ we distinguish the following cases:

- The non-degenerate case, in which both $f$ and $g$ are Lipschitz continuous up to zero.

The degenerate case is divided into

- The $f$-degenerate case, in which $f$ is only locally Lipschitz continuous in $\mathbf{R}^{+}$;

- The $g$-degenerate case, in which $g$ is only locally Lipschitz continuous in $\mathbf{R}^{+}$.

Before we give the proof of Theorem 2.3 we first consider the following lemma.

Lemma 2.5. Let the hypotheses $H($ i)-(iii) be satisfied and let $\left(u_{i}, v_{i}\right), i=1,2$, be as in Theorem 2.3. Further, let $\eta_{1}, \eta_{2} \in \mathbf{R}$ such that $v_{1}\left(\eta_{1}\right)=v_{2}\left(\eta_{2}\right) \in(0,1)$. Then

$$
u_{1}\left(\eta_{1}\right)=u_{2}\left(\eta_{2}\right)
$$

Proof. We give the proof in terms of the original dependent variable, thus

$$
\begin{aligned}
u^{\prime} & =-c_{1} f(1-u) g(v) \\
v^{\prime} & =c_{2}(v-u)
\end{aligned}
$$


in $\mathbf{R}$. Define the shifted functions

$$
\tilde{u}_{2}(\eta)=u_{2}\left(\eta+\eta_{2}-\eta_{1}\right)
$$

and

$$
\tilde{v}_{2}(\eta)=v_{2}\left(\eta+\eta_{2}-\eta_{1}\right)
$$

for all $\eta \in \mathbf{R}$. Clearly this pair defines again a travelling wave for $(\mathrm{BC})$ with the same wavespeed $c$, see Proposition 1.1. By assumption we have $v_{1}\left(\eta_{1}\right)=\tilde{v}_{2}\left(\eta_{1}\right) \in(0,1)$.

First we note that

$$
u_{1}\left(\eta_{1}\right), \tilde{u}_{2}\left(\eta_{1}\right)>0
$$

For if $u_{1}\left(\eta_{1}\right)=0$, for example, then also $u_{1}^{\prime}\left(\eta_{1}\right)=0$ and (2.2a) implies $v_{1}\left(\eta_{1}\right)=0$, a contradiction.

If $u_{1}\left(\eta_{1}\right)=1$, then also $\tilde{u}_{2}\left(\eta_{1}\right)=1$. We show this as follows. Let $\tilde{u}_{2}\left(\eta_{1}\right)<1$ and let

$$
\eta_{3}=\sup \left\{\eta>\eta_{1} \mid u_{1}(\eta)>\tilde{u}_{2}(\eta)\right\} \quad\left(\eta_{3}=+\infty, \text { possibly }\right)
$$

Further let

$$
u(\eta)=\tilde{u}_{2}(\eta)-u_{1}(\eta) \quad \text { and } \quad v(\eta)=v_{1}(\eta)-\tilde{v}_{2}(\eta)
$$

Testing equation (2.2b) for $u$ and $v$ with $v^{+}$gives

$$
\frac{1}{2}\left(v^{+^{2}}\right)^{\prime} \leq c_{2}\left(v^{+^{2}}+u^{+} v^{+}\right)=c_{2} v^{+^{2}} \text { on }\left[\eta_{1}, \eta_{3}\right)
$$

Using $v^{+}\left(\eta_{1}\right)=0$ and Gronwall's Lemma we obtain

$$
v^{+}=0 \quad \text { in }\left(\eta_{1}, \eta_{3}\right) \text {. }
$$


From equation (2.2a) we now deduce

$$
u^{\prime}=-c_{1}\left\{f\left(1-\bar{u}_{2}\right) g\left(\tilde{v}_{2}\right)-f\left(1-u_{1}\right) g\left(v_{1}\right)\right\} \leq 0 \quad \text { in }\left[\eta_{1}, \eta_{3}\right) \text {. }
$$

Integrating this gives

$$
0=u\left(\eta_{3}\right) \leq u\left(\eta_{1}\right)<0
$$

a contradiction.

Hence we may now suppose without loss of generality

$$
1>u_{1}\left(\eta_{1}\right) \geq \tilde{u}_{2}\left(\eta_{1}\right)>0
$$

and show below that

$$
u_{1}\left(\eta_{1}\right)>\bar{u}_{2}\left(\eta_{1}\right)
$$

leads to a contradiction.

For the differences $u$ and $v$ we obtain from (2.2)

$$
\begin{aligned}
u^{\prime} & =-c_{1}\left\{f\left(1-\tilde{u}_{2}\right) g\left(\tilde{v}_{2}\right)-f\left(1-u_{1}\right) g\left(v_{1}\right)\right\} \\
& =-c_{1}\left\{\left[f\left(1-\bar{u}_{2}\right)-f\left(1-u_{1}\right)\right] g\left(\tilde{v}_{2}\right)+f\left(1-u_{1}\right)\left[g\left(\tilde{v}_{2}\right)-g\left(v_{1}\right)\right]\right\}
\end{aligned}
$$

and

$$
v^{\prime}=c_{2}(v+u)
$$

in $\mathbf{R}$. Testing the corresponding equations for $u$ and $v$ with $u^{+}$and $v^{+}$ respectively gives

$$
\begin{aligned}
\frac{1}{2}\left(u^{+^{2}}\right)^{\prime} & =-c_{1}\left\{f\left(1-\tilde{u}_{2}\right)-f\left(1-u_{1}\right)\right\} g\left(\tilde{v}_{2}\right) u^{+}- \\
& -c_{1} f\left(1-u_{1}\right)\left\{g\left(\tilde{v}_{2}\right)-g\left(v_{1}\right)\right\} u^{+}
\end{aligned}
$$

and

$$
\frac{1}{2}\left(v^{+^{2}}\right)^{\prime}=c_{2}\left(v^{+^{2}}+u v^{+}\right)
$$


in R. By (2.3) and the monotonicity we have

$$
u_{1}, \tilde{u}_{2} \leq u_{1}\left(\eta_{1}\right)<1 \quad \text { on }\left(\eta_{1},+\infty\right)
$$

This allows us to use the Lipschitz continuity of $f$ in the whole interval $\left[\eta_{1},+\infty\right)$. For $g$, however, we have to select a sufficiently small right neighborhood of $\eta_{1}$, say $\left(\eta_{1}, \eta_{1}+\delta\right)$, where $v_{1}, \tilde{v}_{2}>0$ and consequently $g$ is Lipschitz continuous. Hence there exist constants $L_{1}, L_{2}\left(L_{1}\right.$ independent of $\delta$ ), such that

$$
\frac{1}{2}\left(u^{+^{2}}\right)^{\prime} \leq\left\{L_{1} u^{+^{2}}+L_{2} u^{+} v^{+}\right\}
$$

and

$$
\frac{1}{2}\left(v^{+^{2}}\right)^{\prime} \leq c_{2}\left\{v^{+^{2}}+u^{+} v^{+}\right\}
$$

in $\left(\eta_{1}, \eta_{1}+\delta\right)$. By Gronwall's inequality and $u^{+}\left(\eta_{1}\right)=v^{+}\left(\eta_{1}\right)=0$ we obtain

$$
u^{+}=v^{+}=0 \quad \text { in }\left[\eta_{1}, \eta_{1}+\delta\right] .
$$

If $g$ is non-degenerate, the argument holds for arbitrary $\delta>0$ and therefore

$$
u^{+}=v^{+}=0 \quad \text { in }\left[\eta_{1},+\infty\right) \text {. }
$$

If $g$ degenerates the argument holds in the interval $\left[\eta_{1}, \eta^{*}\right]$, where $\eta^{*}$ is the first zero of $v_{1}$.

Now suppose $v_{1}\left(\eta^{*}\right)=0$. Hence $v_{1}^{\prime}\left(\eta^{*}\right)=0$, and equation (2.2b) implies $u_{1}\left(\eta^{*}\right)=0$. We also have (from $(2.2 a)$ )

$$
u^{\prime} \leq 0 \quad \text { in }\left[\eta_{1}, \eta^{*}\right]
$$

By assumption we have $u\left(\eta_{1}\right)<0$, and thus $u<0$ in $\left[\eta_{1}, \eta^{*}\right]$. Thus in particular $u\left(\eta^{*}\right)<0$, implying $\bar{u}_{2}\left(\eta^{*}\right)<u_{1}\left(\eta^{*}\right)=0$, a contradiction.

Thus we may conclude that

$$
u^{+}=v^{+}=0 \quad \text { in }\left[\eta_{1},+\infty\right)
$$


in both cases, and also

$$
u^{\prime} \leq 0 \quad \text { in }\left[\eta_{1},+\infty\right)
$$

Integrating this equation contradicts the boundary conditions at $\eta=+\infty$.

We are now in the position to prove Theorem 2.3.

Proof of Theorem 2.3. By Lemma 2.5 there exist points $\eta_{0}, \eta_{1} \in$ R. such that the transformed wave

$$
\bar{u}_{2}(\eta)=u_{2}\left(\eta+\eta_{0}\right) \quad \text { and } \quad \tilde{v}_{2}(\eta)=v_{2}\left(\eta+\eta_{0}\right) \quad \text { for } \eta \in \mathbf{R}
$$

coincides with $u_{1}$ and $v_{1}$ at the point $\eta_{1}$, i.e.

$$
u_{1}\left(\eta_{1}\right)=\tilde{u}_{2}\left(\eta_{1}\right)=: \bar{u} \quad \text { and } \quad v_{1}\left(\eta_{1}\right)=\tilde{v}_{2}\left(\eta_{1}\right)=: \bar{v}
$$

where $\bar{u}, \bar{v} \in(0,1)$. By the uniqueness of the initial value problem for equations (2.2) with Lipschitz continuous right-hand side there exist $\underline{d}, \bar{d}>0$ such that

$$
u_{1}-\tilde{u}_{2}=v_{1}-\tilde{v}_{2} \equiv 0 \quad \text { in }\left(\eta_{1}-\underline{d}, \eta_{1}+\bar{d}\right) \text {. }
$$

In the non-degenerate case obviously $\underline{d}=\bar{d}=+\infty$. In the degenerate case, however, the argument holds only if $f\left(1-u_{1}\left(\eta_{1}-\underline{d}\right)\right)>0$ and $g\left(v_{1}\left(\eta_{1}+\bar{d}\right)\right)>0$. We therefore distinguish the cases, where $\eta_{1}-\underline{d}$ and $\eta_{1}+\bar{d}$ are the first zeros of $f\left(1-u_{1}(\cdot)\right)$ and $g\left(v_{1}(\cdot)\right)$, respectively.

- $u_{1}\left(\eta_{1}-\underline{d}\right)=1$. Then we continue the solution with the unique solution of

$$
v^{\prime}=c_{2}(v-1) \quad \text { for } \eta<\eta_{1}-\underline{d},
$$

where $v\left(\eta_{1}-\underline{d}\right)$ follows from the construction.

$\bullet v_{1}\left(\eta_{1}+\bar{d}\right)=0$. Then we continue the solution as $(0,0)$ for $\eta>\eta_{1}+\bar{d}$. 
Thus we may conclude

$$
u_{1}-\tilde{u}_{2}=v_{1}-\tilde{v}_{2} \equiv 0 \quad \text { in } \mathbf{R}
$$

for both the degenerate and non-degenerate case. This proves the assertion.

\section{FINITENESS}

Referring to the properties of the orbit as mentioned in section 2, we investigate here the existence of a conversion front (where $u=1$ and the solid is fully converted) and a penetration front (where $u=v=0$ and no reaction has yet taken place).

In the following we suppose that $\{u, v, c\}$ is a travelling wave in the sense of Corollary 1.2 where $f$ and $g$ satisfy $H(i)-(i i i)$.

Definition 3.1. A travelling wave has a conversion front if

$$
a:=\inf \{\eta \mid u(\eta)<1\}>-\infty
$$

and has a penetration front if

$$
b:=\sup \{\eta \mid u(\eta)>0\}<+\infty
$$

Remark 3.2. As a direct consequence of the existence and uniqueness we have

$$
v(\eta)<1 \quad \text { for all } \eta \in \mathbf{R} \text {. }
$$

Next let

$$
\tilde{b}=\sup \{\eta \mid v(\eta)>0\} .
$$

Then $\tilde{b}=b$. This follows from the observations

$$
\begin{aligned}
b<+\infty & \Rightarrow u(\eta)=0 \text { for } \eta \geq b \\
& \Rightarrow v(\eta)=0 \text { for } \eta \geq b \quad \text { (from equation (2.2a)) } \\
& \Rightarrow \tilde{b} \leq b
\end{aligned}
$$


and

$$
\begin{aligned}
\tilde{b}<+\infty & \Rightarrow v(\eta)=0 \text { for } \eta \geq \tilde{b} \\
& \Rightarrow u(\eta)=0 \text { for } \eta \geq \tilde{b} \quad \text { (from equation (2.2b)) } \\
& \Rightarrow b \leq \tilde{b} .
\end{aligned}
$$

We have the following characterization of the existence of a conversion front.

\section{Theorem 3.3.}

$$
a>-\infty \Leftrightarrow \frac{1}{f} \in L^{1}(0, \delta)
$$

for some $\delta \in(0,1)$.

Proof. ' $\Rightarrow$ '. We first estimate equation (2.2a) by

$$
u^{\prime}=-c_{1} f(1-u) g(v) \geq-c_{1} f(1-u) g(1) .
$$

For any $a<\eta_{1}<\eta_{2}$ we obtain

$$
\int_{\eta_{1}}^{\eta_{2}} \frac{-u^{\prime}}{f(1-u)} d \eta \leq c_{1} g(1)\left(\eta_{2}-\eta_{1}\right)
$$

or

$$
\int_{1-u\left(\eta_{1}\right)}^{1-u\left(\eta_{2}\right)} \frac{1}{f(z)} d z \leq c_{1} g(1)\left(\eta_{2}-\eta_{1}\right) .
$$

Letting $\eta_{1} \searrow a$ yields

$$
\int_{0}^{1-u\left(\eta_{2}\right)} \frac{1}{f(z)} d z \leq c_{1} g(1)\left(\eta_{2}-a\right)<+\infty .
$$

Thus

$$
\frac{1}{f} \in L^{1}(0, \delta)
$$


for some $\delta>0$.

$' \xi^{\prime}$. We now prove that $a>-\infty$ by contradiction. Thus suppose $a=-\infty$. This implies

$$
u(\eta)<1 \quad \text { for all } \eta \in \mathbf{R} \text {. }
$$

\section{Writing}

$$
-\frac{u^{\prime}}{f(1-u)}=c_{1} g(v)
$$

and integrating this expression from $\eta_{1}$ to $\eta_{2}$ for arbitrary $\eta_{1}<\eta_{2}$ with $v\left(\eta_{2}\right)>0$ gives

$$
\begin{gathered}
\int_{\eta_{1}}^{\eta_{2}} \frac{-u^{\prime} d \eta}{f(1-u)}=\int_{1-u\left(\eta_{1}\right)}^{1-u\left(\eta_{2}\right)} \frac{d z}{f(z)}= \\
=c_{1} \int_{\eta_{1}}^{\eta_{2}} g(v(\eta)) d \eta \geq c_{1} g\left(v\left(\eta_{2}\right)\right)\left(\eta_{2}-\eta_{1}\right),
\end{gathered}
$$

where $g\left(v\left(\eta_{2}\right)\right)>0$. Letting $\eta_{1} \rightarrow-\infty$ leads to a contradiction.

Remark 3.4. If $f \in C^{0,1}\left(\mathbf{R}_{0}^{+}\right)$then $\frac{1}{f} \notin L^{1}(0, \delta)$ and consequently $a=-\infty$. If $f(z)=z^{p}$ with $0<p<1$ then $\frac{1}{f} \in L^{1}(0, \delta)$ for all $\delta \in(0,1)$, which implies the existence of a conversion front.

Next we characterize the existence of a penetration front.

Theorem 3.5. Suppose $g$ is concave in $(0, \delta)$ for some $\delta>0$, then

$$
b<+\infty \Leftrightarrow \frac{1}{\sqrt{G}} \in L^{1}(0, \delta),
$$

where

$$
G(v)=\int_{0}^{v} g(z) d z
$$


Proof. Differentiating equation (2.2b) with respect to $\eta$ and substituting the result into (2.2a) leads to the expression

$$
v^{\prime \prime}=c_{2}\left(v^{\prime}+c_{1} f(1-u) g(v)\right) \quad \text { in } \mathbf{R} \text {. }
$$

Because $v^{\prime} \leq \mathbf{0}$ in $\mathbf{R}$ and $f$ is non-decreasing we can estimate the second derivative of $v$ by

$$
v^{\prime \prime} \leq c_{1} c_{2} f(1) g(v) \quad \text { in } \mathbf{R} .
$$

Multiplying this inequality by $v^{\prime}$ and integrating the result from $\eta$ to $+\infty$ yields, using $v(+\infty)=v^{\prime}(+\infty)=0$,

$$
v^{\prime}(\eta)^{2} \leq 2 c_{1} c_{2} f(1) \int_{0}^{v(\eta)} g(z) d z \quad \text { in } \mathbf{R}
$$

and hence

$$
v^{\prime}(\eta) \geq-\bar{K} \sqrt{G(v(\eta))} \quad \text { in } \mathbf{R},
$$

where $\vec{K}$ is the positive constant given by

$$
\bar{K}=\sqrt{2 c_{1} c_{2} f(1)}
$$

We also want to construct an upper bound for $v^{\prime}$ which has the same structure as the right hand side of (3.2). To obtain this upper bound we substitute the lower bound (3.2) into equation (3.1) and find

$$
v^{\prime \prime} \geq c_{2}\left(-\bar{K} \sqrt{G(v)}+c_{1} f(1-u) g(v)\right) \quad \text { in } \mathbf{R} \text {. }
$$

Now define $\eta_{1}$ such that

$$
f(1-u(\eta))>\bar{f} \quad \forall \eta>\eta_{1},
$$


where $\bar{f}$ is a given constant in $(0, f(1))$. Multiplying inequality (3.3) by $v^{\prime}$ and integrating the result from $\eta \geq \eta_{1}$ to $+\infty$ gives, again using $v^{\prime}(+\infty)=v(+\infty)=0$,

$\left(v^{\prime}(\eta)\right)^{2} \geq-2 c_{2} \bar{K} \int_{0}^{\nu(\eta)} \sqrt{G(z)} d z+2 c_{1} c_{2} \bar{f} \int_{0}^{v(\eta)} g(z) d z \quad$ in $\left[\eta_{1},+\infty\right)$

We now define $\eta_{2}$ by $v\left(\eta_{2}\right)=\delta$. The concavity of $g$ in $(0, \delta)$ implies

$$
v(\eta) \leq \frac{\delta}{g(\delta)} g(v(\eta)) \quad \text { in }\left[\eta_{2},+\infty\right)
$$

and the monotonicity of $g$ that

$$
\int_{0}^{v} g(z) d z \leq v g(v)
$$

Thus (3.6) and (3.7) imply

$$
G(v(\eta))=\int_{0}^{v(\eta)} g(z) d z \leq \frac{\delta}{g(\delta)} g(v(\eta))^{2} \quad \text { in }\left[\eta_{2},+\infty\right)
$$

or

$$
\sqrt{G(v(\eta))} \leq C(\delta) g(v(\eta)) \quad \text { in }\left[\eta_{2},+\infty\right)
$$

where

$$
C(\delta)=\sqrt{\frac{\delta}{g(\delta)}} .
$$

Setting $\bar{\eta}=\max \left\{\eta_{1}, \eta_{2}\right\}$ and substituting (3.8) into (3.5) gives

$$
\left(v^{\prime}(\eta)\right)^{2} \geq\left(-2 c_{2} \bar{K} C(\delta)+2 c_{1} c_{2} \bar{f}\right) \int_{0}^{v(\eta)} g(z) d z \quad \text { in }[\bar{\eta},+\infty)
$$

We now proceed as follows: 
1. Suppose $g^{\prime}(0+)=+\infty$. Then $C(\delta) \searrow 0$ as $\delta \searrow 0$ and we can choose $\delta$ sufficiently small so that

$$
2 c_{1} c_{2} \bar{f}-2 c_{2} \bar{K} C(\delta)>0,
$$

which yields

$$
\left(v^{\prime}(\eta)\right)^{2} \geq \underline{K}^{2} G(v(\eta)) \quad \text { in }[\bar{\eta},+\infty),
$$

or

$$
v^{\prime}(\eta) \leq-\underline{K} \sqrt{G(v(\eta))} \quad \text { in }[\bar{\eta},+\infty)
$$

with

$$
\underline{K}=\sqrt{2 c_{1} c_{2} \bar{f}-2 c_{2} \bar{K} C(\delta)} .
$$

Combining the bounds (3.2) and (3.10) we find

$$
-\bar{K} \leq \frac{v^{\prime}}{\sqrt{G(v)}} \leq-\underline{K} \quad \text { in }[\bar{\eta},+\infty)
$$

We integrate this expression from $\eta_{1}$ to $\eta_{2}$ with $\eta_{1}<\eta_{2}$ and $\eta_{1}, \eta_{2} \epsilon$ $[\bar{\eta},+\infty)$. This gives

$$
\underline{K}\left(\eta_{2}-\eta_{1}\right) \leq \int_{v\left(\eta_{2}\right)}^{v\left(\eta_{1}\right)} \frac{d z}{\sqrt{G(z)}} \leq \bar{K}\left(\eta_{2}-\eta_{1}\right) .
$$

From this expression the statement of the theorem immediately follows.

2. Suppose $g^{\prime}(0+)<+\infty$. Then $g \in C^{0,1}\left(\mathbf{R}_{0}^{+}\right)$and a local uniqueness argument near $v=0$ shows that no finite wave can exist in this case. It remains to show that

$$
\frac{1}{\sqrt{G}} \notin L^{1}(0, \delta)
$$

This follows from (3.9). We have

$$
\frac{1}{\sqrt{G}} \geq \frac{1}{C(\delta) g(v)} \notin L^{1}(0, \delta)
$$


as a consequence of the differentiability.

When $\frac{1}{\sqrt{G}} \in L^{1}(0, \delta)$ we have a precise result about the behaviour of $v$ near the point $b$.

Theorem 3.6. Let $g$ be as in Theorem 3.5 and let $\{u, v, c\}$ denote a travelling wave with a penetration front. Define $w: \mathbf{R} \rightarrow \mathbf{R}_{0}^{+}$by

$$
w(\eta)=\int_{0}^{v(\eta)} \frac{d z}{\sqrt{G(z)}}
$$

Then

$$
w^{\prime}(\eta)>-\sqrt{2 c_{1} c_{2} f(1)} \quad \text { for } \eta<b
$$

and

$$
w^{\prime}(\eta) \rightarrow-\sqrt{2 c_{1} c_{2} f(1)} \quad \text { for } \eta \rightarrow b-
$$

Proof. Multiplying equation (3.1) by $v^{\prime}$ and integrating the result fron $\eta$ to $b$ with $\eta<b$ yields

$$
-\frac{1}{2} v^{\prime}(\eta)^{2}=c_{2} \int_{\eta}^{b} v^{\prime}(s)^{2} d s+c_{1} c_{2} \int_{\eta}^{b} f(1-u(s)) g(v(s)) v^{\prime}(s) d s
$$

for $\eta \in(-\infty, b)$. We divide this expression by $G$ and obtain

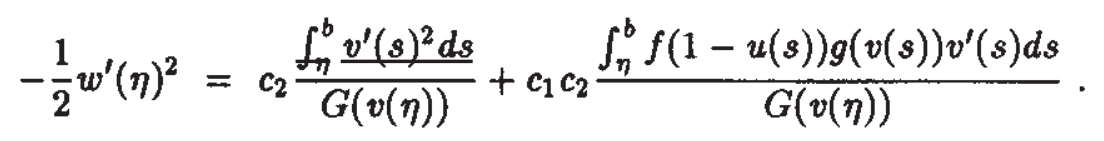

Now add $c_{1} c_{2} f(1)$ to both sides. This gives

$$
\begin{aligned}
& -\frac{1}{2} w^{\prime}(\eta)^{2}+c_{1} c_{2} f(1)=c_{2} \frac{\int_{\eta}^{b} v^{\prime}(s)^{2} d s}{G(v(\eta))}+ \\
& +c_{1} c_{2} \frac{f_{\eta}^{b}\{f(1-v(s))-f(1)\} g(v(s)) v^{\prime}(s) d s}{G(v(\eta))}
\end{aligned}
$$




$$
=: A_{1}+A_{2}
$$

for $\eta \in(-\infty, b)$. Since $A_{1}+A_{2}>0$ the desired inequality for $w^{\prime}$ follows inmediately. It remains to show that $A_{1}+A_{2} \rightarrow 0$ as $\eta \rightarrow b-$. Using inequality (3.2) in the expression for $A_{1}$ results in

$$
A_{1} \leq 2 c_{1} c_{2}^{2} f(1) \frac{f_{\eta}^{b} G(v(s)) d s}{G(v(\eta))} \leq 2 c_{1} c_{2}^{2} f(1)(b-\eta) \quad \text { in }(-\infty, b)
$$

and thus $A_{1} \rightarrow 0$ as $\eta \rightarrow b-$. To show that $A_{2} \rightarrow 0$ we first choose $\epsilon>0$. The continuity of $f$ and $u$ and the fact $u(b)=0$ yields the existence of $\eta_{\epsilon}<b$ such that

$$
f(1)-f(1-u(\eta))<\epsilon
$$

for all $\eta \in\left(\eta_{\epsilon}, b\right)$. This means

$$
A_{2} \leq c_{1} c_{2} \epsilon
$$

from which the result follows.

\section{LIMIT CASE $D \searrow 0$}

We study here the behaviour of the travelling waves as they approach the limit $D \searrow 0$. Then the influence of dispersion and diffusion vanishes.

Theorem 4.1. Suppose $H(i)$-(iii) are satisfied. Let $\left\{D_{n}\right\}_{n=1}^{\infty}$ be a sequence of positive numbers for which $D_{n} \searrow 0$ as $n \rightarrow \infty$. For each $n \in \mathrm{N}$, let $\left\{u_{n}, v_{n}, c\right\}$ denote a travelling wave corresponding to $D=D_{n}$ and the same boundary conditions (BC), which has been translated such that $u_{n}(0)=\tilde{u}$ for some $\tilde{u} \in(0,1)$. Then there exist functions $u, v: \mathbf{R} \rightarrow[0,1]$ and a subsequence $n_{l} \rightarrow \infty$ such that for all $K \subset \subset \mathbf{R}$

$$
\begin{array}{ll}
u_{n_{l}} \rightarrow u & \text { pointwise on } \mathbf{R} \text { and in } C(K) \\
v_{n_{1}} \rightarrow v & \text { a.e. on } \mathbf{R} \text { and in } L^{q}(K) \text { for all } q \geq 1 .
\end{array}
$$


for $n_{l} \rightarrow \infty$. Moreover we have

$$
u=v \quad \text { in } \mathbf{R}
$$

and $u$ satisfies the problem

$$
(P)\left\{\begin{array}{l}
u \in C^{1}(\mathbf{R}) \\
u^{\prime}=-c_{1} f(1-u) g(u) \text { in } \mathbf{R} ; \\
u(-\infty)=1, u(+\infty)=0 .
\end{array}\right.
$$

Proof. Introducing the constants $c_{1}=\frac{1}{c S^{*}}$ and $c_{3}=\frac{\lambda c S^{*}}{C^{*}}$ in equations (1.9) yields

$$
\begin{aligned}
u^{\prime} & =-c_{1} f(1-u) g(v) \\
v^{\prime} & =\frac{c_{3}}{D}(v-u)
\end{aligned}
$$

in R. Note that both $c_{1}$ and $c_{3}$ are independent of $D$. Since $0 \leq u_{n} \leq$ $1,0 \leq v_{n} \leq 1,-c_{1} f(1) g(1) \leq u_{n}^{\prime} \leq 0$ and $v_{n}^{\prime} \leq 0$ for all $n \in \mathrm{N}$, we obtain that

$$
\left\|u_{n}\right\|_{\infty, \mathbf{R}}, \quad\left\|u_{n}^{\prime}\right\|_{\infty, \mathbf{R}}, \quad\left\|v_{n}\right\|_{\infty, \mathbf{R}} \text { and }\left\|v_{n}^{\prime}\right\|_{1, \mathbf{R}}
$$

are uniformly bounded with respect to $n$. Applying the Arzela-Ascoli Theorem to the sequence $\left\{u_{n}\right\}_{n=1}^{\infty}$ and using the compact embedding $W^{1,1}(K) \hookrightarrow L^{q}(K)$ with $1 \leq q<\infty$ for the sequence $\left\{v_{n}\right\}_{n=1}^{\infty}$ gives that there exist functions $u \in C(\mathbf{R}) \cap W^{1, \infty}(\mathbf{R})$ and $v \in L^{\infty}(\mathbf{R})$ such that

$$
0 \leq u, v \leq 1 \quad \text { on } \mathbf{R}
$$

and a subsequence $n_{l} \rightarrow \infty$ (denoted here again by $n$ ) such that

$$
u_{n} \rightarrow u \quad \text { in } C(K)
$$


and

$$
v_{n} \rightarrow v \quad \text { in } L^{q}(K)
$$

for all $K \subset \subset \mathbf{R}$. Equation (4.1b) gives

$$
\left\|v_{n}-u_{n}\right\|_{1, \mathbf{R}}=\frac{D_{n}}{c_{3}}\left\|v_{n}^{\prime}\right\|_{1, \mathbf{R}} \rightarrow 0 \quad \text { as } n \rightarrow \infty
$$

from which it follows that ( $v$ possibly redefined on a set of measure zero)

$$
u=v \quad \text { in } \mathbf{R} \text {. }
$$

We now conclude that

$$
f\left(1-u_{n}\right) g\left(v_{n}\right) \rightarrow f(1-u) g(u) \quad \text { a.e. in } \mathbf{R} .
$$

Using this in equation (4.1a) for $\left(u_{n}, v_{n}\right)$ and passing to the limit for $n \rightarrow \infty$ leads to

$$
u \in C^{1}(\mathbf{R})
$$

and

$$
u^{\prime}=-c_{1} f(1-u) g(u) \quad \text { in } \mathbf{R} .
$$

It remains to verify the boundary conditions for $u$. The monotonicity of $u$ and the normalization $u_{n}(0)=u(0)=\tilde{u} \in(0,1)$ implies that $u(-\infty) \in[\tilde{u}, 1]$ and $u(+\infty) \in[0, \tilde{u}]$. This excludes the trivial limits $u \equiv 0$ and $u \equiv 1$. The proof that indeed $u(-\infty)=1$ and $u(+\infty)=0$ is given in detail by [vDK].

Corollary 4.2. The function $u$ from Theorem 4.1 satisfies

(i) If $a:=\inf \{\eta \in \mathbf{R} \mid u(\eta)<1\}$, then $a>-\infty \Leftrightarrow \frac{1}{f} \in L^{1}(0, \delta)$ for some $\delta>0$;

(ii) If $b:=\sup \{\eta \in \mathbf{R} \mid u(\eta)>0\}$, then $b<+\infty \Leftrightarrow \frac{1}{g} \in L^{\mathbf{1}}(0, \delta)$ for some $\delta>0$.

Theorem 4.3. Let $\left\{u_{D}, v_{D}, c\right\}$ be a monotone non-increasing 
travelling wave satisfying boundary conditions $(B C)$ and let $u$ be a monotone non-increasing solution of Problem $(P)$. Further let $\chi:[0,1] \rightarrow \mathbf{R}^{+}$ be defined by

$$
\chi(u)=c_{1} f(1-u) g(u)
$$

and suppose there exists $\vec{u} \in(0,1)$ such that $\chi$ is non-decreasing in $(0, \tilde{u})$ and non-increasing in $(\bar{u}, 1)$. In addition suppose that $g$ is concave in $(0,1)$ and if $g$ degenerates in zero, then also assume

$$
\frac{g(z)}{z} \in L^{1}(0,1)
$$

If the functions $u_{D}$ and $u$ are normalized such that $u_{D}(0)=u(0)=\tilde{u}$, then

$$
\left\|u_{D}-u\right\|_{\infty, \mathbb{R}} \leq D \frac{c_{1}}{c_{3}} f(1) L_{i}
$$

and

$$
\left\|v_{D}-v\right\|_{\infty, \mathbf{R}} \leq D \frac{c_{1}}{c_{3}}\left\{f(1) L_{i}+f(1-\tilde{u}) g(\tilde{u})\right\}
$$

for all $D>0$ and $i=1,2$. We use here $i=1$ to denote the nondegenerate case with

$$
L_{1}=g^{\prime}(0)
$$

and $i=2$ to denote the $g$-degenerate case with

$$
L_{2}=\int_{0}^{1} \frac{g(z)}{z} d z
$$

Proof. We write equation (4.1b) as

$$
v_{D}=\frac{D}{c_{3}} v_{D}^{\prime}+u_{D} \quad \text { in } \mathbf{R}
$$

and substitute this expression in equation (4.1a) to obtain

$$
u_{D}^{\prime}=-c_{1} f\left(1-u_{D}\right) g\left(\frac{D}{c_{3}} v_{D}^{\prime}+u_{D}\right) \geq-\chi\left(u_{D}\right) \text { in } \mathbf{R},
$$


where we used the monotonicity of $g$ and $v_{D}^{\prime} \leq 0$. For $u$ we have the equation

$$
u^{\prime}=-\chi(u) \quad \text { in } \mathbf{R} .
$$

Taking the difference of these equations and testing with $\left(u-u_{D}\right)^{+}$gives

$$
\frac{1}{2}\left\{\left(u-u_{D}\right)^{+^{2}}\right\}^{\prime} \leq-\left(\chi(u)-\chi\left(u_{D}\right)\right)\left(u-u_{D}\right)^{+} \quad \text { in } \mathbf{R} .
$$

Since $u, u_{D}<\tilde{u}$ in $\mathbf{R}^{+}$, the monotonicity of $\chi$ in $(0, \tilde{u})$ implies

$$
\frac{1}{2}\left\{\left(u-u_{D}\right)^{+^{2}}\right\}^{\prime} \leq 0 \quad \text { in } \mathbf{R}^{+}
$$

With $u(0)=u_{D}(0)$ this gives

$$
u \leq u_{D} \quad \text { in } \mathbf{R}^{+}
$$

Testing with $\left(u-u_{D}\right)^{-}$gives by the same arguments

$$
u \geq u_{D} \quad \text { in } \mathbf{R}^{-}
$$

Thus we have

$$
\tilde{u}>u_{D} \geq u \Rightarrow \chi(u) \leq \chi\left(u_{D}\right) \text { in } \mathbf{R}^{+}
$$

and

$$
u \geq u_{D}>\tilde{u} \Rightarrow \chi(u) \leq \chi\left(u_{D}\right) \text { in } \mathbf{R}^{-} .
$$

At points where $u, u_{D}$ and $v_{D}$ are simultaneously positive we have

$$
\begin{aligned}
\left(u_{D}-u\right)^{\prime} & =\chi(u)-c_{1} f\left(1-u_{D}\right) g\left(\frac{D}{c_{3}} v_{D}^{\prime}+u_{D}\right) \\
& \leq \chi\left(u_{D}\right)-c_{1} f\left(1-u_{D}\right) g\left(\frac{D}{c_{3}} v_{D}^{\prime}+u_{D}\right) \\
& =\chi\left(u_{D}\right)\left\{1-\frac{g\left(\frac{D}{c_{3}} v_{D}^{\prime}+u_{D}\right)}{g\left(u_{D}\right)}\right\}
\end{aligned}
$$




$$
=\chi\left(u_{D}\right) H\left(\frac{D}{c_{3}} v_{D}^{\prime}\right)
$$

where

$$
H(\alpha)=1-\frac{g\left(\alpha+u_{D}\right)}{g\left(u_{\bar{D}}\right)} .
$$

Because $g$ is concave we have for $\alpha<0$

$$
\frac{g\left(\alpha+u_{D}\right)}{\alpha+u_{D}} \geq \frac{g\left(u_{D}\right)}{u_{D}}
$$

and thus

$$
H(\alpha) \leq-\frac{\alpha}{u_{D}} .
$$

Hence (4.4) can be estimated further to give

$$
\left(u_{D}-u\right)^{\prime} \leq-\chi\left(u_{D}\right) \frac{D}{c_{3}} \frac{v_{D}^{\prime}}{u_{D}}=-D \frac{c_{1}}{c_{3}} f\left(1-u_{D}\right) \frac{q\left(u_{D}\right)}{u_{D}} v_{D}^{\prime} .
$$

We distinguish the following cases: If $g^{\prime}\left(0^{+}\right)<\infty$, then the concavity implies

$$
\frac{g(z)}{z} \leq g^{\prime}(0)
$$

which gives in (4.5)

$$
\left(u_{D}-u\right)^{\prime} \leq-D \frac{c_{1}}{c_{3}} f(1) g^{\prime}(0) v_{D}^{\prime}
$$

From this we deduce

$$
\begin{gathered}
\left(u_{D}-u\right)(\eta) \leq D \frac{c_{1}}{c_{3}} f(1) g^{\prime}(0)\left(v_{D}(0)-v_{D}(\eta)\right) \leq \\
\leq D \frac{c_{1}}{c_{3}} f(1) g^{\prime}(0) \text { for } \eta>0
\end{gathered}
$$

and 


$$
\begin{gathered}
\left(u-u_{D}\right)(\eta) \leq D \frac{c_{1}}{c_{3}} f(1) g^{\prime}(0)\left(v_{D}(\eta)-v_{D}(0)\right) \leq \\
\leq D \frac{c_{1}}{c_{3}} f(1) g^{\prime}(0) \text { for } \eta<0,
\end{gathered}
$$

which gives the desired estimate for $\left\|u_{D}-u\right\|_{\infty, \mathbf{R}}$ in the non-degenerate case. obtain

If $g^{\prime}\left(0^{+}\right)=\infty$, we use again the concavity of $g$ and $v_{D} \leq u_{D}$ to

$$
\frac{g\left(u_{D}\right)}{u_{D}} \leq \frac{g\left(v_{D}\right)}{v_{D}}
$$

which results in

$$
\left(u_{D}-u\right)^{\prime} \leq-D \frac{c_{1}}{c_{3}} f\left(1-u_{D}\right) \frac{g\left(v_{D}\right)}{v_{D}} v_{D}^{\prime}
$$

This leads to

$$
\begin{aligned}
& \left(u_{D}-u\right) \leq D \frac{c_{1}}{c_{3}} f(1) \int_{v(\eta)}^{v(0)} \frac{g(z)}{z} d z \leq \\
& \leq D \frac{c_{1}}{c_{3}} f(1) \int_{0}^{1} \frac{g(z)}{z} d z \quad \text { for } \eta>0,
\end{aligned}
$$

which is finite because $\frac{g(z)}{z}$ is integrable as was assumed and

$$
\begin{gathered}
\left(u-u_{D}\right) \leq D \frac{c_{1}}{c_{3}} f(1) \int_{v(0)}^{v(\eta)} \frac{g(z)}{z} d z \leq \\
\leq D \frac{c_{1}}{c_{3}} f(1) \int_{0}^{1} \frac{g(z)}{z} \quad \text { for } \eta<0 .
\end{gathered}
$$

This gives the desired inequality for $\left\|u-u_{D}\right\|_{\infty}, \mathbf{R}$ in the degenerate case.

To obtain the result for $\left\|v-v_{D}\right\|_{\infty, \mathbf{R}}$ we solve equation (4.1b) which yields

$$
v_{D}(\eta)=\frac{c_{3}}{D} \int_{\eta}^{\infty} u_{D}(t) \exp \left(\frac{c_{3}}{D}(\eta-t)\right) d t
$$


and thus

$$
v_{D}(\eta)-v(\eta)=v_{D}(\eta)-u(\eta)=\frac{c_{3}}{D} \int_{\eta}^{\infty} u_{D}(t) \exp \left(\frac{c_{3}}{D}(\eta-t)\right) d t-u(\eta)
$$

in $\mathbf{R}$. Hence

$$
\left|v_{D}(\eta)-v(\eta)\right| \leq \frac{c_{3}}{D} \int_{\eta}^{+\infty}\left|u_{D}(t)-u(\eta)\right| \exp \left(\frac{c_{3}}{D}(\eta-t)\right) d t \leq I_{1}+I_{2}
$$

where

$$
I_{1}=\frac{c_{3}}{D} \int_{\eta}^{\infty}\left|u_{D}(t)-u(t)\right| \exp \left(\frac{c_{3}}{D}(\eta-t)\right) d t \leq D \frac{c_{1}}{c_{3}} f(1) L_{\dot{q}}
$$

and

$$
I_{2}=\frac{c_{3}}{D} \int_{\eta}^{\infty}\{u(\eta)-u(t)\} \exp \left(\frac{c_{3}}{D}(\eta-t)\right) d t
$$

Equation (4.1a) and the structure of $\chi$ yields

$$
u(\eta)-u(t) \leq \chi(\tilde{u})(t-\eta)
$$

implying

$$
I_{2} \leq \frac{D}{c_{3}} \chi(\tilde{u})
$$

Finally we combine (4.6) and the inequalities for $I_{1}$ and $I_{2}$ to obtain the second estimate of the theorem.

Example 4.4. Let $f(z)=z^{p}$ and $g(z)=z^{m}$ with $p>0$ and $0<m<1$. The function $g$ is concave and degenerates in zero. We find $L_{2}=1 / m$, and the estimates become

$\left\|u_{D}-u\right\|_{\infty, \mathbf{R}} \leq D \frac{c_{1}}{c_{3} m} \quad$ and $\quad\left\|v_{D}-v\right\|_{\infty, \mathbf{R}} \leq D \frac{c_{1}}{c_{3}}\left(\frac{1}{m}+\frac{m}{m+p}\right)$ 
Remark 4.5. The function

$$
g(z)=\frac{-1}{\log z}
$$

satisfies all the conditions in Theorem 4.3, except for (4.3). This shows the necessity of $(4.3)$ in the degenerate case.

Acknowledgements. While working on this problem, the first author enjoyed the hospitality of the Institute of Mathematics and its Applications at Minnesota and the second author enjoyed the hospitality of the Koninklijke/ Shell-Laboratory at Amsterdam.

\section{References.}

[vDK] Duijn, C.J. van and Knabner, P. Solute transport in porous media with equilibrium and non-equilibrium multiple-site adsorption: Travelling waves, J. reine angewandte Math. 415, (1991), 1-49.

[DS] Díaz, J.I. and Stakgold, I. Mathematical analysis of the conversion of a porous solid by a distributed gas reaction, Proc. XI CDEYA/I Congr. Matematica Aplicada, Málaga (1989), 217-223.

[FB] Froment, G.F. and Bischoff, K.B. Chemical reactor design and analysis, Wiley and Sons, (1979).

[LMS] Liddo, A.Di, Maddalena, L. and Stakgold, I. Travelling waves for distributed gas-solid reactions, To appear in J. Diff. Eq.

[S] Stakgold, I., Partial extinction in reaction-diffusion, Conferenze del seminario di matematica, 224, Bari (1987). 
[SES] Szekeley, J., Evans, J.W. and Sohn, H.Y. Gas-Solid reactions, Academic Press, (1976).

TU Delft,

Recibido: 12 de febrero de 1993

Department of Mathematics

P.O. BOX 5031,

NL-2600 GA Delft

The Netherlsnds

Leiden University

Matbematical Institute

P.O. Box 9512,

NL-2300 RA Leiden

The Netherlands. 